\title{
A Proposed Mentoring Program to Raise Achievement Motivation During Covid-19 Pandemic for Elementary Students in South Sharqiyah, Oman
}

\author{
Rashid Saif Rashid Almaqhousi \\ Faculty of Education, International Islamic University of Malaysia (IIUM), Selangor, Malaysia \\ rashid303020@gmail.com
}

KEYWORDS: $\quad$ Counseling, Achievement Motivation, Covid-19, Blended Learning.

Crossref doi https://doi.org/10.51345/.v32i3.450.g236

\begin{abstract}
:
This article discusses a proposed counseling program to raise achievement motivation in light of the Corona pandemic (Covid-19) and blended education among students of basic and post-basic education in the Governorate of South Al Sharqiyah in the Sultanate of Oman. The article is part of a research project carried out by the researcher in government schools affiliated with the Ministry of Education, with the aim of building a proposed indicative program suitable for training students on study habits and skills, which contribute to raising the achievement motivation among students. The study sought to identify the components of the counseling program based on training in studying skills, study habits that contribute to raising achievement motivation and raising students' achievement levels. The (proposed) program was designed for the extension program, based on previous studies and theoretical literature. The researcher benefited from the study of Abu Alia (1993); Al-Sawalmeh (2001); and Tarawneh (2005). Where the program (10) consisted of: A demonstration for a proposed period of time between (15 to 25) minutes. It aims to meet the first acquaintance and clarify building relationships, and the second session to introduce students to the concept. The situation is under observation. As for the third academic meetings, they aimed to discuss skills: managing time during the lecture, taking notes during the lecture, remembering and forgetting how to review courses, how to prepare for the exam, and dealing with care with the exam. And suggested many appropriate guiding methods, to achieve the objectives of the pilot program, which represents: (dialogues, debate, modeling, cooperative education, brainstorming, feedback, emergency management, homework, instructions, silent reading, relaxation). The researcher recommends applying this program of counseling proposed by the counseling and psychological counseling specialist, psychologist and social worker in schools to students of basic education and post-basic education in the Governorate of South Al Sharqiah in the Sultanate of Oman and psychological and academic counseling centers in Omani public and private universities and colleges.
\end{abstract}

\section{REFERENCES:}

Allam, S. et. al., (2020). Online Distance Learning Readiness During Covid-19 Outbreak Among Undergraduate Students. Journal of Academic Research in Business and Social Sciences ,10(5), 642-657.

Allyn, P. Bacon. (2004. Motivating Students to Learn, www.Quik.com.

Pajares, F. (2002). Self-Efficacy Beliefs in Academic Contexts. An outline, URL: www.emory.edu/Education/mfp/efftalk.html. 


\title{
برنامج إرشاد مقترح لرفع دافعية الإنجاز في ضوء جائحة كوفيد-19 لطلبة التعليم الأساسي وبعد الأساسي في محافظة جنوب الشرقية بسلطنة عمان
}

\author{
راشد بن سيف بن راشد المقحوصي

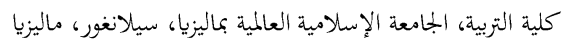 \\ rashid303020@gmail.com
}

الكلمات المفتاحية | ل الإرشاد، دافعية الإنجاز، كوفيد-19، التعليم المدمج.

Crossref doi $\quad$ https://doi.org/10.51345/.v32i3.450.g236

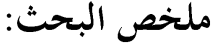

يناقش هذا المقال برنامج إرشاد مقترح لرفع دافعية الإنجاز في ضوء جائحة كوفيد-19 والتعليم المدمج لدى طلبة التعليم الاساسي والتعليم ما بعد الاساسي في محافظة جنوب الشرقية بسلطنة عمان. المقال جزء من مشروع بحثي يجريه الباحث في المدارس الحكومية التابعة لوازرة التربية والتعليم، بجدف بناء برنامج إرشادي مقترح يصلح لتدريب الطلبة على العادات والمهارات الدراسية، التي تساهم في رفع دافعية الإنجاز لدى الطلبة. سعت الدراسة إلى تحديد مكونات البرنامج الإرشادي القائم على التدريب على مهارات المذاكرة، العادات الدراسية التي تساهم في رفع دافعية الإنجاز وفي رفع المستويات التحصيلية لدى الطلبة. تم تصميم برنامج الصيغة (المقترحة) للبرنامج الإرشادي، استناد إلى دراسات سابقة والأدب النظري. وقد استفاد الباحث من دراسة أبو علياء (1993)؛ السوالمة (2001)؛ والطراونة (2005). حيث تالف البرنامج (10) من: عرض توضيحي لفترة زمنية مقترحة بين (15 إلى 25) دقيقة. يهدف إلى لقاء التعارف الأول وتوضيح بناء العلاقات، والدورة الثانية لتعريف الطلاب بالمفهوم. الوضع تحت الملاحظة. أما الاجتماعات الأكاديمية الثالثة كانت تمدف التداول حول مهارات: إدارة الوقت اثناء الخحاضرة، تدوين الملاحظات اثناء المحاضرة، تذكر ونسيان كيفية مراجعة الدورات، كيفية التحضير للامتحان، التعامل باهتمام مع الامتحان. واقترحت العديد من الأساليب الإرشادية المناسبة، لتحقيق أهداف البرنامج التجريبي، الذي يمثل: (حوارات، مناظرة، نمذجة، تعليم تعاوين، عصف ذهني، تغذية راجعة، إدارة الطوارئ، الواجبات المنزلية، التعليمات، القراءة الصامتة، الاسترخاء). يوصي الباحث بتطبيق هذا البرنامج الإرشاد المقتح من قبل اخصائي التوجيه والارشاد النفسي والاخصائي النفسي والاخصائي الاجتماعي في المدارس على الطلبة التعليم الاساسي والتعليم ما بعد الاساسي في محافظة جنوب الشرقية بسلطنة عمان ومراكز الإرشاد النفسي والأكاديمي في الجامعات والكليات العمانية الحكومية والخاصة.

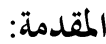

يعد التحصيل العلمي محورا هاما من محاور الاهتمام الأساسي في التعليم المدرسي، ويشكل مستوى تحصيل

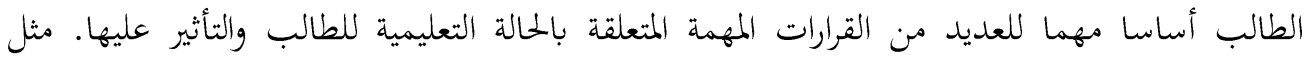


الاستمرار في الدراسة ومعدل التحصيل، أو تغيير عادات الدراسة ومهارات الدراسة، المتعلقة بالمستوى الدراسي الذي حققه الطالب متمثلا في مستوى التحصيل، وتنظر المؤسسات التعليمية إلى مستوى التحصيل المرتبط به، على أنه: الوسيلة الرئيسية المتاحة لتحديد مستوى كفاءة الطالب وقدرته، والتنبؤ بقدرته على النجاح في دراساةم المستقبلية(1). ولا تشير مستويات التحصيل المرتفعة أو المنخفضة لدى الطلاب بالضرورة إلى تمييز أو ضعف فئف في قدراتم

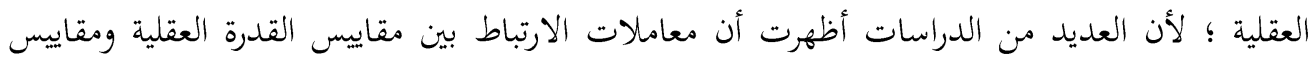
التحصيل تتراوح (30 ، . _ 0.80). وهذا يدل على وجود علاقة تباين في مستويات الإنجاز تحددها عوامل القدرة العقلية، كما يعني أن هناك عوامل أخرى غير تلك القدرات تفسر نسبة كبيرة من التباين في التحصيل. الذكاء ضروري للإنجاز، لكنه لا يكفي بحد ذاته للنجاح الأكاديمي (2).

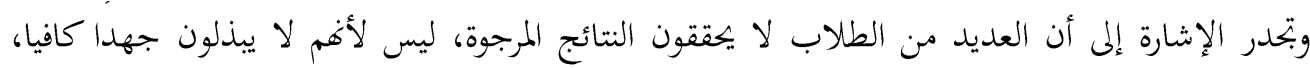

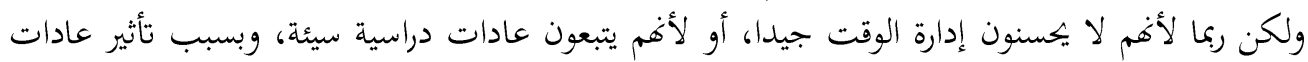

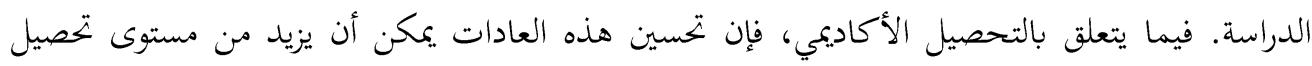

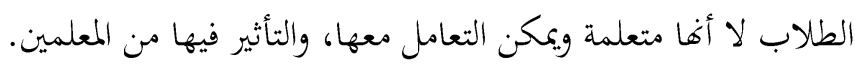

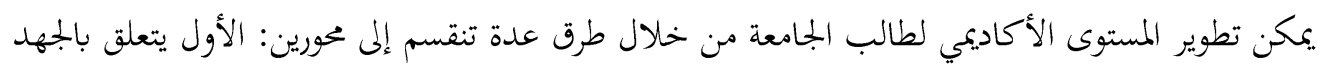

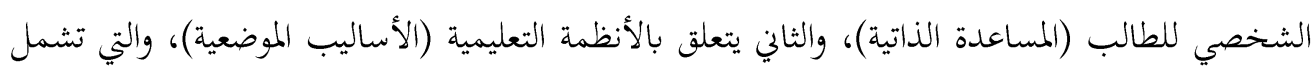
نظاما جديدا للقبول، وطرق تدريس حديثة، وطرق مثلى للتقييم والامتحانات. إلى جانب العوامل

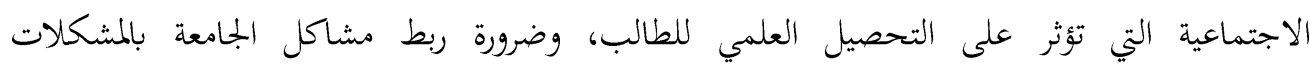
الاجتماعية(3) الاجنماعة التية تملي التطورات العالمية استخدام التكنولوجيا، بما في ذلك التعلم الإلكتروني، في بحال التعليم والتدريس، لكنها تعاني من عقبات تقف في طريق تطويرها واعتمادها في المؤسسات التعليمية(4). وفي سعي المؤسسات

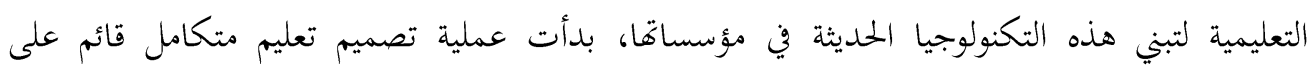

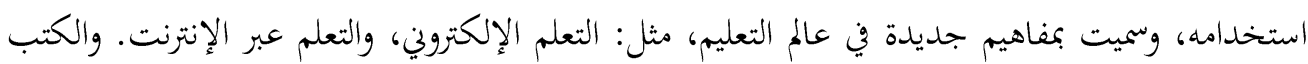
الإلكترونية والمكتبة الإلكترونية والوسائط الإلكترونية الأخرى(5).

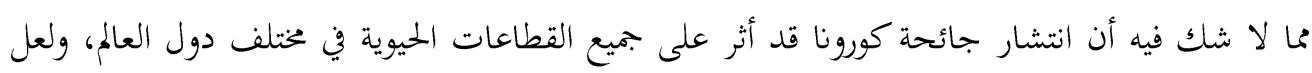
أبرز هذه القطاعات هو قطاع التعليم الذي كان من أكثر القطاعات تضررا، حيث كان التفاعل بين الأطراف. للعملية التعليمية محورية في النظام التعليمي، وقد أوصت اليونسكو بضرورة اللجوء إلى التعليم عن 
بعد باستخدام التطبيقات الذكية التي تحولت إلى منصات تعليمية في عصر فيروس كورونا، وهذا يعني أن التقنيات الحديثة أصبحت إلزامية شريك في عملية التعلم التربوي(6). رغم كل الممارسات التي صاحبت جائحة كورونا (كوفيد_19) وفي مقدمتها دفع عجلة التعليم عن بعد والتعلم الإلكتروني إلى أمام، إلا إها تركت في الوقت نفسه أكبر تعطيل للأنظمة التعليمية في التاريخ؛ مع

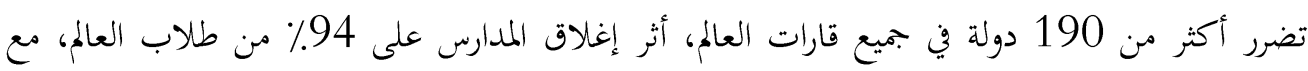

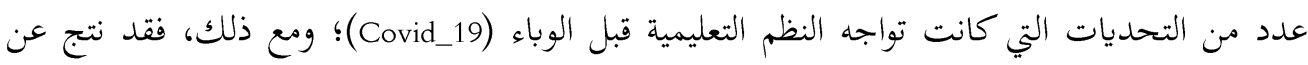

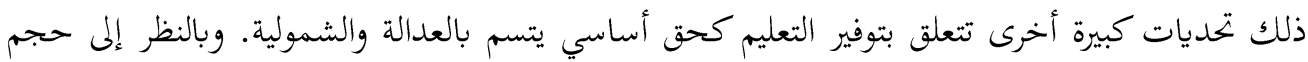

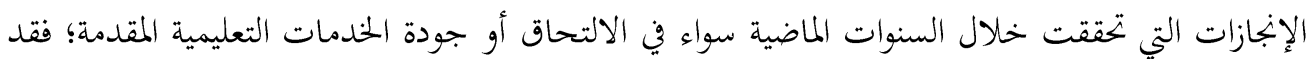
كشف الوباء عن عدة تحديات أبرزها: استمرار التدريس والتعلم أثناء إغلاق المدارس، والخسارة التعليمية نتيجة لذلك أو نتيجة عمليات التعليم عن بعد، وهذا بدوره سيصبح واضحا في المراحل الأولى من التعليم، نظرا لوجود عدد محدود من الطلاب ضمن إطار محفز وبيئة تفاعلية اجتماعية مدرسية غنية(7). البرنامج الإرشادي هو مجموعة من الأنشطة والتدريبات والتجارب التي تمارسها المدرسة والأجهزة التربوية

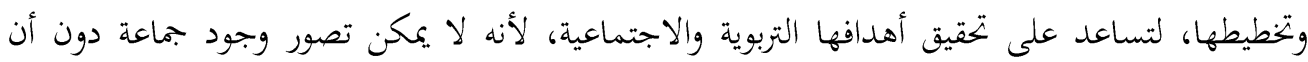
يتضمن وجودها برنامج تخطط وتنفذه وتتابعه وتقيم نتائجه، مما يبرز كجهاز تفاعل تربوي واجتماعي (8). ويعرف الباحث البرنامج الإرشادي المستخدم في هذه الدراسة بأنه: مجموعة من الفنيات والإجراءات والأساليب المنظمة، والمخططة لها بصورة علمية تطبق على إفراد الفئة المستهدفة. ولإلقاء الضوء على هذا الموضوع، يسعى الباحث إلى طرح توصيات تساهم في تخطى هذه التحديات والاستمرار في التحصيل العلمي وتفادي قلة الإنجاز، وعدم الحصول على النسبة المئوية المناسبة. وكذلك إلى بناء برنامج إرشادي مقترح لرفع مستويات دافعية الإنجاز لدى طلبة التعليم الاساسي والتعليم ما بعد الاساسي في محافظة جنوب الشرقية بسلطنة عمان.

\section{مشكلة الدراسة :}

يشهد العالم حاليا حدثًا كبيرا قد يهدد التعليم بأزمة هائلة، ربما تكون الأكثر خطورة في عصرنا المعاصر.

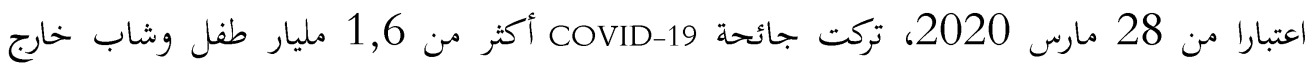
المدرسة في 161 دولة، ما يقرب من 80٪ من الطلاب مسجلين في المدارس على مستوى العالم. جاء مناء

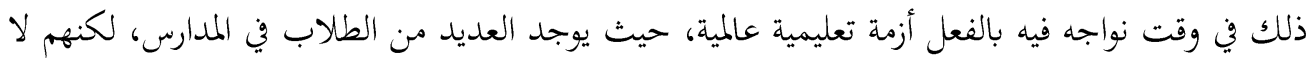

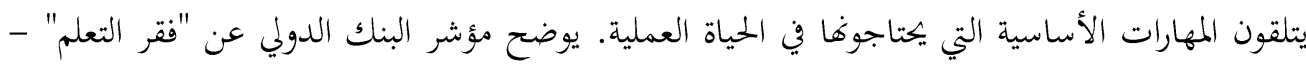


أو نسبة الطلاب الذين لا يستطيعون القراءة أو الفهم في سن العاشرة - أن نسبة هؤلاء الأطفال في البلدان المنخفضة والمتوسطة الدخل قبل تفشي الفيووس كانت 53 ٪. إذا لم نتحرك، فقد يؤدي هذا الوباء إلى تفاقم هذه النتيجة(9). وتشير الدراسات إلى أن طلاب المدارس يفقدون ثلث ما يجب أن يتعلموه إذا لم يتم التخاذ الإجراءات التصحيحية، ونتيجة لذلك يتركون المدرسة عندما يصلون إلى الصف العاشر، أو لا يكتسبون المهارات المطلوبة، وتقدر منظمة اليونسكو أن قد يؤدي الأثر الاقتصادي للوباء وحده إلى توقف 23.8 مليون طفل ئل وشاب إضافي من التعليم قبل الابتدائي إلى التعليم العالي(10). ولتسليط الضوء على هذه المشكلة، ظهرت بعض المبادرات الدولية للحد من آثارها، من خلال عقد المد ندوات وعقد اجتماعات وزارية، لعل أبرزها ندوة اليونسكو الإقليمية بعنوان "الضياع التعليمي في ظل

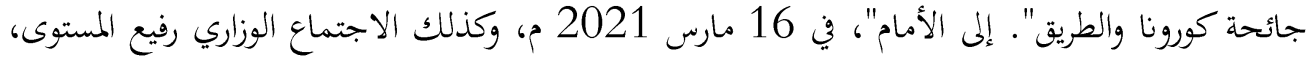
الذي نظمته اليونسكو في 29 مارس 2021، بعنوان "عام واحد بعد COVID-19، مع إعطاء الأولوية

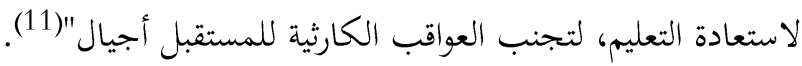

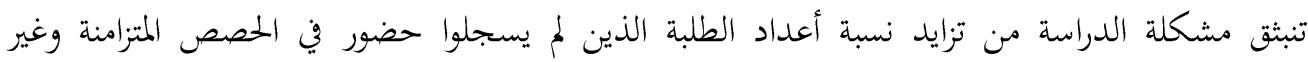
المتزامنة، كجزء من التعليم المدمج الذي فرضته جائحة كرونا (كوفيد_19)، حيث تشير الإحصائيات إلى مرئ إن إعداد الطلبة الذين لم يسجلوا حضور في الحصص المتزامنة وغير المتزامنة في الجزء الاول من العام الدراسي 2021/2020م وقد زاد عدد هؤلاء الطلبة في الجزء الثاني من نفس العام الدراسي. وهذه تعني من وجهة نظر الباحث ارتفاع نسبة الطلبة الذين لم يتمكنوا من الحضور وهذا ما يؤثر سلبا على أداء الطالب الدراسي والمستوى العلمي في مرحلة التعليم ما بعد الاساسي، وقد يؤدي ذلك إلى الانخفاض يي الدافعية للإنجاز إن لم تتخذ الإجراءات اللازمة فهذا سيؤدي إلى زيادة معدل الفاقد التعليمي إضافة إلى الخسائر التي قد تلحق بالطالب من الناحية المادية والنفسية جراء نظرة المجتمع له. إضافة إلى ذلك فان وضع الطالب في المؤسسة التعليمية وفقده التدريجي للمستوى التعليمي. بتعله يدخل في مرحلة من القلق بسبب العبء الدراسي المتراكم عليه ومن ثم زيادة معدل الفاقد في الدروس وشعورة على عدم مقدرته للمتابعة دروسه لاحقا. وتشير العديد من الدراسات (سليمان، 1990 فئه ؛ اللبابيدي،

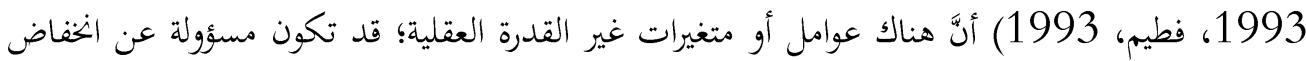
أداء الطلاب، وأن هناك متغيرات مثل عادات الدراسة ومهارات الدراسة التي قد تلعب دورا مهما في صنع هذه الاختلافات والاختلافات في التحصيل الدراسي بين الطلاب أثناء وجودهم في المدرسة. 
وعند محاولة الباحث مسح الدراسات التي أجريت على طلبة الجامعة في سلطنة عمان فلم يتسن للباحث حسب حدود علمه واطلاعه العثور على أية دراسة هدفت إلى بناء برنامج إرشادي لرفع دافعية الإنجاز.

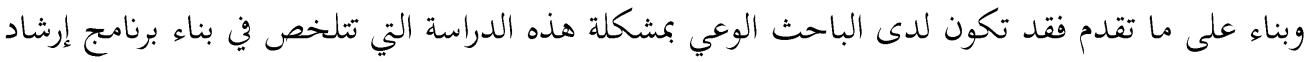
مقترح قائم على التدريب على العادات والمهارات الدراسية وذلك لرفع دافعية الإنجاز لدى طلبة التعليم الاساسي والتعليم ما بعد الاساسي في محافظة جنوب الشرقية بسلطنة عمان

\section{أهداف الدراسة:}

تمدف هذه الدراسة إلى: بناء برنامج إرشادي مقترح يصلح لتدريب الطلبة على العادات والمهارات الدراسية، التي قد تساهم في رفع دافعية الإنجاز لدى طلبة التعليم الاساسي والتعليم ما بعد الاساسي في محافظة جنوب الشرقية بسلطنة عمان.

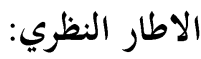

\section{دافعية الإنجاز}

معظم الدوافع المتعلقة بالتحصيل هي دافع الإنجاز، والذي حظي باهتمام كبير من قبل العديد من الباحثين، ويعود الفضل في تقديم الدراسة لهنري موراي (Henry Murray)، الذي يعتبر أول من أدخل مفهوم (الحاجة

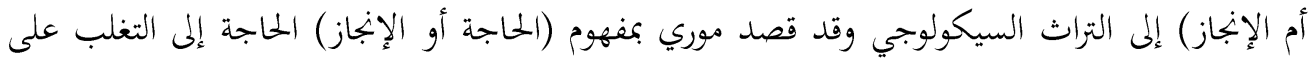
الصعوبات، وممارسة التحكم، والسعي للقيام بشيء صعب بطريقة مرضية وسريعة، وتحقيق مستويات عالية من التميز والتنافس مع الآخرين. لذلك فإن الإنجاز من المصادر المهمة التي تؤثر على إنجازات المتعلم. قد يؤثر دافع الطالب الضعيف سلبا على تحصيله، حتى لو كان طالبا ذكيا. من هنا يمكن استنتاج مستوى دافع الإنجاز من مستوى الإنجاز (2001; Pajares and Schunk ). تعد عادات الدراسة من أكثر المتغيرات المساهمة في توقع النجاح الأكاديمي، ويشير فريمان وموريس (Freeman and Morss;1993) أن الطلاب الذين يستخدمون طرقا معينة مثل الأشكال أو الرسومات أو غيرها من الأساليب التي تساعد تحسين الذاكرة أفضل. الأداء في الاختبارات. 


\section{التعليم في ظل جائحة كورونا: - مان}

بعد عام من الإجراءات الاستثنائية التي اتخذتها دول العالم في ظل جائحة كوفيد 19، ابتهت الأنظمة التعليمية إلى وضع خطط للتعافي وعودة طبيعية مأمولة للعام الدراسي المقبل من خلال تشخيص فقدان التعليم وإيماد حلول وبدائل له. تحسين مخرجات التعلم، ودعم الطلاب الذين لا يمتلكون بعض ولمه المهارات

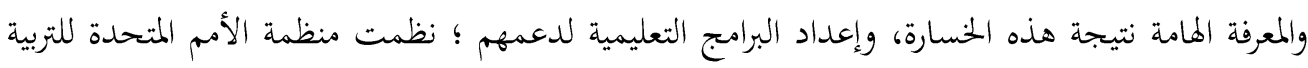
والعلم والثقافة (اليونسكو) اجتماعا وزاريا في مارس 2021 حول إعطاء الأولوية لاستعادة التعليم بعد هذا الوباء، وتناول العديد من الموضوعات أبرزها قضية فقدان التعليم، والحفاظ على فتح المدارس وضماغا. بيئة تعليمية آمنة وتحول رقمي (12). ويشير خبراء تكنولوجيا التعليم إلى أن أهم ما يميز التعلم عن بعد، عن نظم التعليم الأخرى - مثل التعليم التقليدي والتعلم المفتوح - هو المرونة وحرية الاختيار، حيث يتسم التعلم عن بعد بالمرونة في التحرر والتخلص من قيود النظام التقليدي وواجباته، وعدم حضور المتعلم للمؤسسة التعليمية، وعلى الدارسة

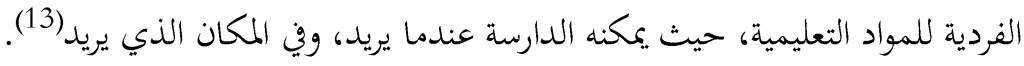
سلطنة عمان ليست بمنأى عما يحدث في كل دول العالم. وقد سارعت السلطنة في الاستجابة لمذه البيانات

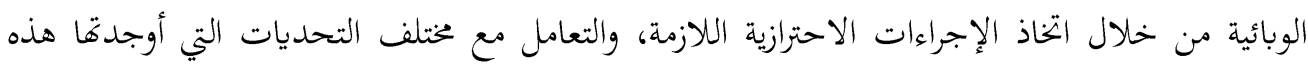

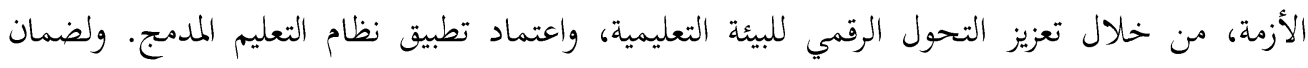
استمرارية العملية التعليمية، فقد تمثلت هذه الإجراءات في الآتي: وضع إطار عام لتشغيل المدارس، يتضمن التهني

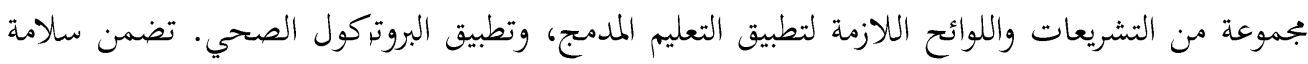
المدرسة والمجتمع التربوي بشكل عام (14). هناك مكاسب عديدة للتعليم في ظل جائحة كورونا منها التحول الرقمي لعمليات التدريس والتقييم، لذلك قمنا بتطبيق ما ترددنا في تطبيقه في الماضي، وتمكين المعلم لقيادة التعلم، وتعزيز الشراكة مع الجمتمع المحلي. المجتمع على نطاق أوسع. في تقويم الطلاب وإتقان التعلم عن بعد والتحفيز والتحفيز لدى الطلاب وابن والتأثير على الجانب النفسي والاجتماعي لهم(15). إعداد المنصات التعليمية والمتوى الرقمي الإلكتروني بالشراكة مع القطاع الخاص، وبتعاون جميع المعلمين

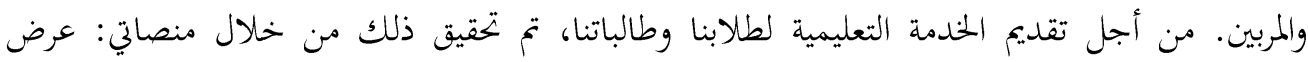
لصفوف الدورة الأولى (1-4)، منصة Google classroom لصفوف الحلقة الثانية والتعليم ما بعد الأساسي مني وحزمة من برامج التدريب عن بعد للهيئات التعليمية والإشرافية والإدارية. لتمكينهم من إيجاد طرق مبتكرة 
للتدريس في هذا العام الاستثنئي. أظهرت مؤشرات الأداء الرقمية الجهود الكبيرة التي يبذها جميع المعلمين والإدارات المدرسية وفنيي تكنولوجيا المعلومات في تفعيل المنصات التعليمية وتوظيفها في توعية طلابنا بما

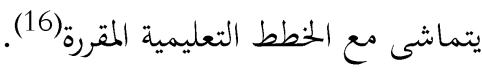

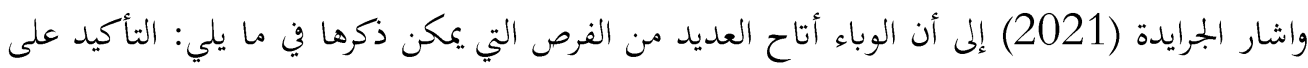
أهمية إيجاد بدائل تعليمية لضمان استمرارية العملية التعليمية، ولفت الانتباه إلى أهمية تطوير وتطوير البنية التحتية التكنولوجية للمؤسسات التعليمية، كما لفت انتباه المعنيين بالتعليم؛ إلى ضرورة وضع معايير لضمان جودة استخدام تقنيات التدريب والمنصات التعليمية، وإلى أهمية دور الأسرة والبيت في تربية أبنائهم، فضلا لإئا عن أهمية تقديم دورات تحدف إلى تطوير التعلم الشخصي والتعليم الرقمي، وضرورة استخدام وتطبيق

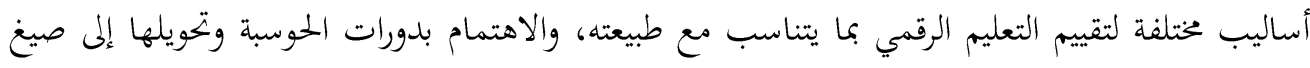

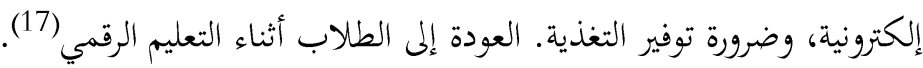

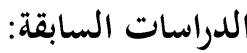

\section{دراسات هدفت إلى بناء برامج إرشادية قائمة على تدريب الطلبة على مهارات المذاكرة}

دراسة سكيميلزر وآخرون (Schmelzer,2000) خلصت إلى أن مسؤولية النجاح والفشل تقع في المقام

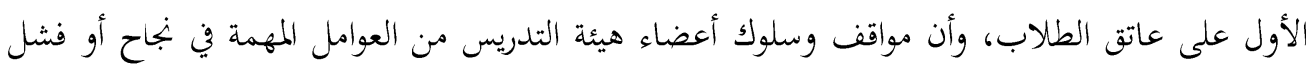
الطلاب. أوصت الدراسة بما يلي: يجب على كل طالب أن يتعلم كيف يدرس بفاعلية، وكيف ينظم وقته دون إهمال الأنشطة الاجتماعية وغيرها، أي عليه أن يتبع أسلوب حياة متوازن. توفير فترات تدريب قصيرة ين مهارات الدراسة الذاتية. عقد دورات تدريبية لأعضاء هيئة التدريس الذين يواجهون صعوبات في المهارات التنظيمية أو غيرها من المهارات المتعلقة بالتدريس. الطراونة، (2005م) أجرى دراسة هدفت إلى استقصاء فعالية برنامج إرشاد عقلاني - انفعالي معرفي في تحسين مستوى فعالية الذات المدركة ودافعية الإنجاز والمستوى التحصيلي لدى طلبة جامعة مؤتة ذوي

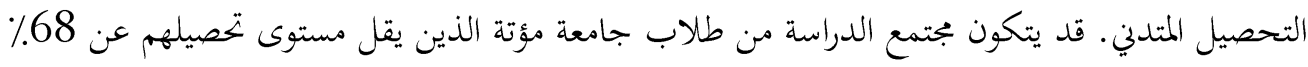

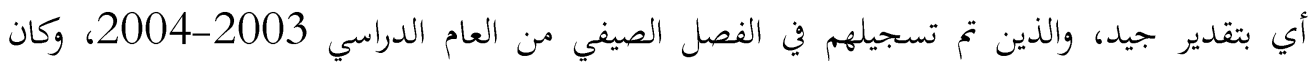

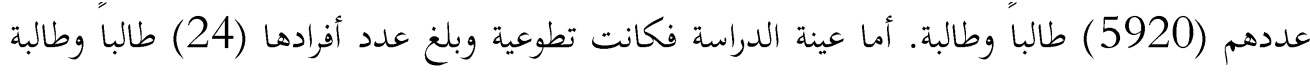

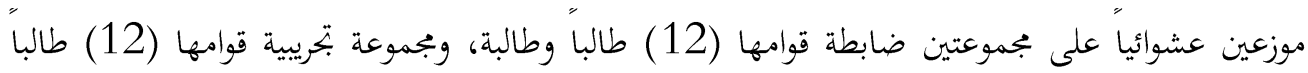


وطالبة. الطلاب. أشارت نتائج الدراسة إلى وجود فروق ذات دلالة إحصائية في الكفاءة الذاتية المسوسة، ودوافع الإنجاز، ومستويات التحصيل بين المجموعة التجريبية والضابطة.

\section{دراسات حول جائحة كرونا ودورها في تغيير التعليم.}

دراسة أبو عباة (2021) بعنوان: "تقييم بحربة المملكة العربية السعودية في التعليم عن بعد في ظل جائحة

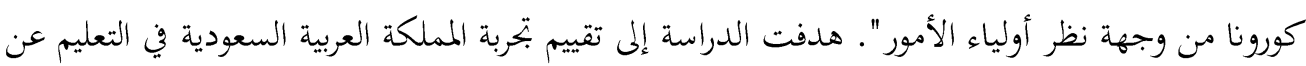
بعد في ظل جائحة كورونا من وجهة نظر أولياء الأمور في مدينة الرياض. واختتمت الدراسة بمجموعة من التوصيات في ضوء النتائج أهمها توجيه إدارات المدارس الابتدائية لزيادة مشاركة أولياء الأمور في الخطط لئه التربوية لأنظمة التعليم عن بعد، وخطة لتدريب المعلمين على نظام التعليم عن بعد. دراسة صافي (2020) واقع استخدام التعليم الإلكتروني الافتراضي بالجامعة الجزائرية في ظل جائحة كورونا، هدفت إلى التعرف على واقع استخدام التعليم الإلكتروني الافتراضي بالجامعة الجزائرية في ظل جائحة كورونا، وطبقت دراسة على عينة من طلبة كلية العلوم الإنسانية والاجتماعية بجامعة العربي

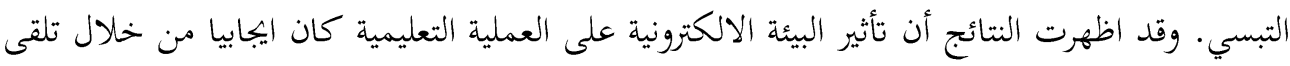

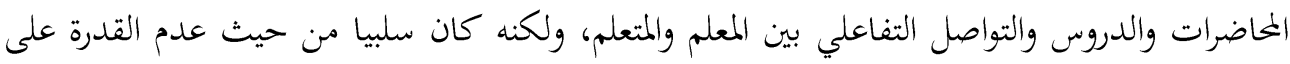

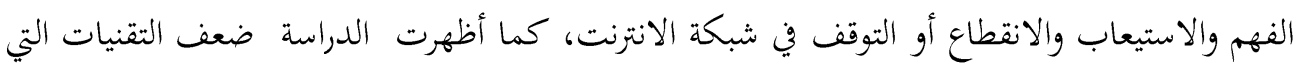
يعتمد عليها في التعليم عن بعد، كذلك هدفت ولت

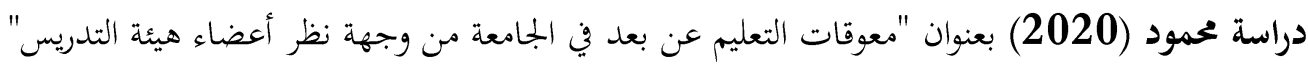
التي هدفت إلى تعرف تطبيقات الذكاء الاصطناعي التي يمكن الإفادة منها في تطوير العملية التعليمية. وقد بـد اعتمدت الدراسة المنهج الوصفي، من خلال استقراء وتحليل الدراسات، تحديات جائحة فيروس كورونا

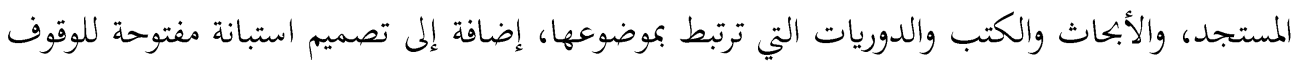
على أهم المشكلات والتحديات التي تواجهها العملية التعليمية، ودور تطبيقات الذكاء الاصطناعي في تركي مواجهة تلك التحديات. وقد تم عرضها على بعض المسئولين عن العملية التعليمية بالتعليم الجامعي وما قبل الجامعي بلغ عددهم (31). وتوصلت الدراسة إلى أنه توجد عدة تحديات ومشكلات تتصل بالجوانب

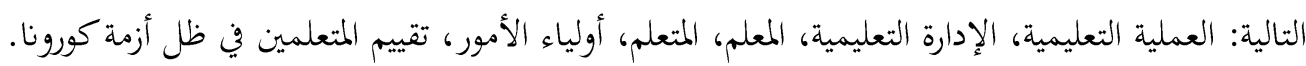
كما توصلت، إلى أنه يمكن توظيف بعض تطبيقات الذكاء الاصطناعي في العملية التعليمية كأنظمة الإداء التعليم الذكي، والمحتوى الذكي، وتقنية الواقع الافتراضي (VR) والواقع المعزز (AR) وغيرها من التطبيقات. 
حسن، (2020) قدم ورقة بحثية تناولت تعليم وتعلم الرياضيات عن بعد، في ظل جائحة كورونا: الواقع

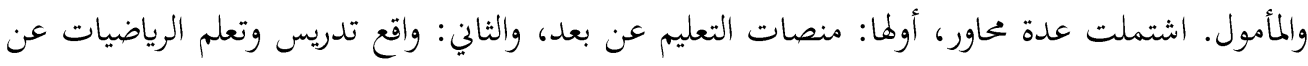

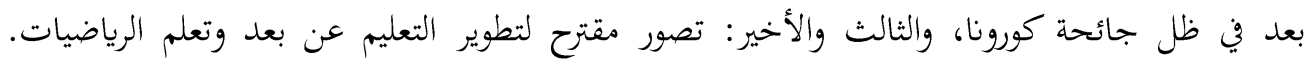

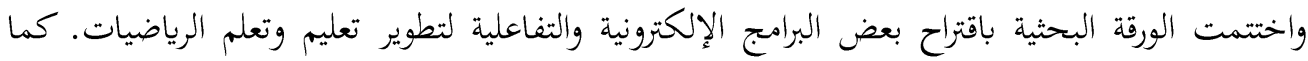

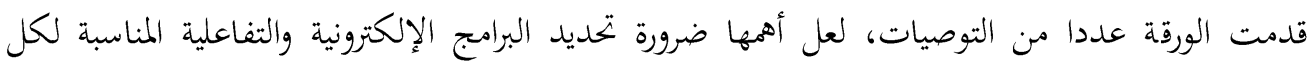

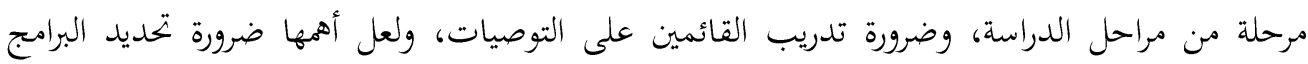

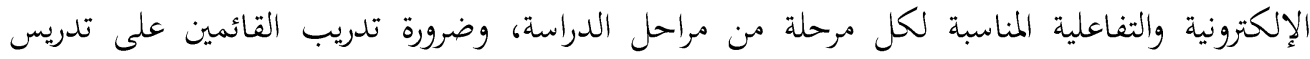

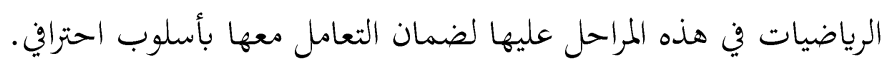

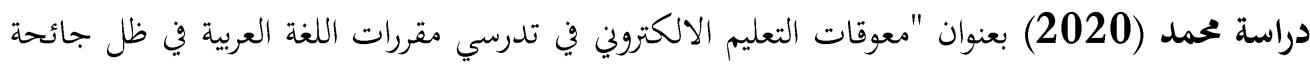

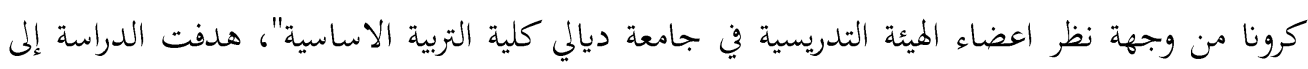

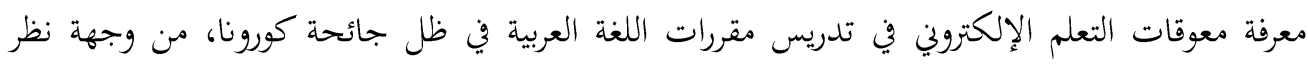

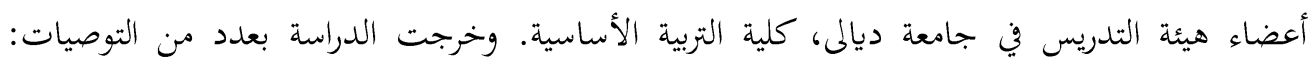

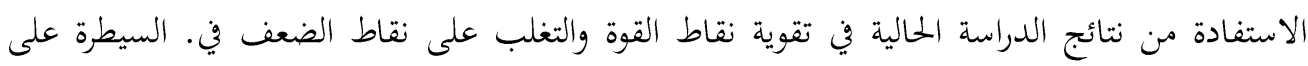

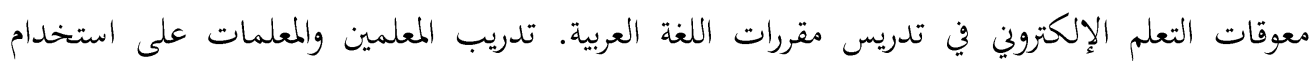

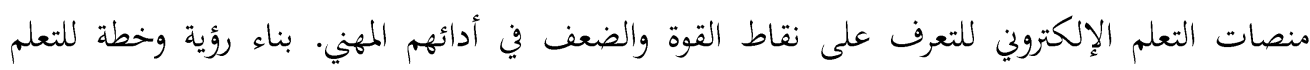

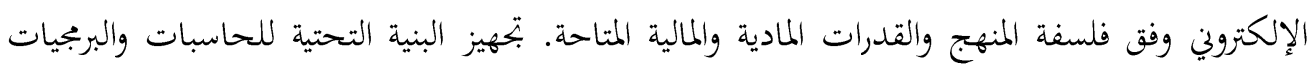
وشبكات الاتصال كالإنترنت والشبكة الخلية. دراسة الظفري، والهانشية، (2020). بعنوان "التحديات الاسرية واستراتيجيات التصدي والنهات لها خلال

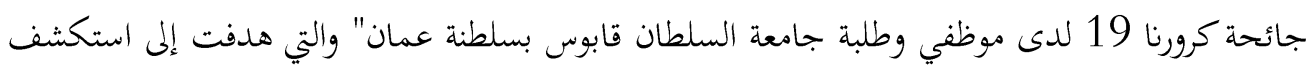

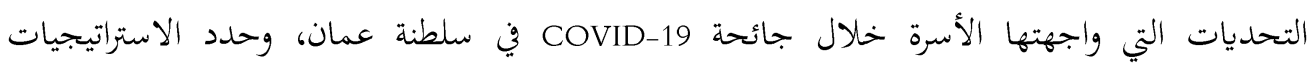

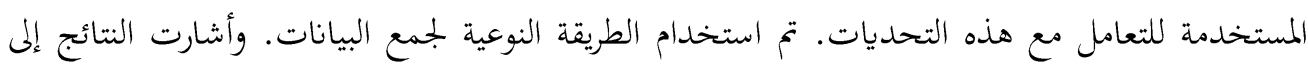

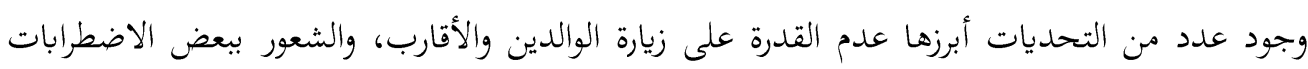

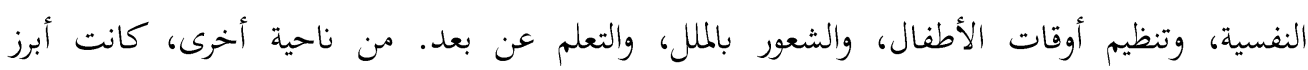

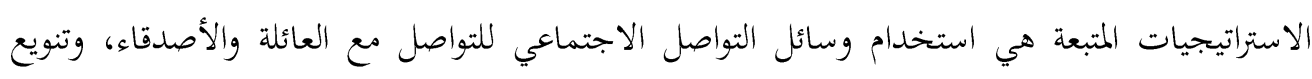

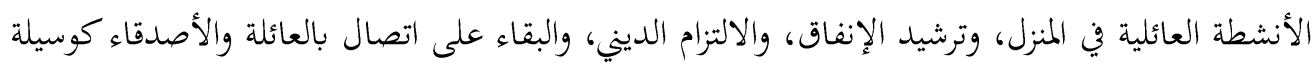
للتكيف النفسي. 
كذلك تناولت الدراسات تقييم التجربة في بيئات تعلم أجنبي دراسة (Allam et. al.,2020) هدفت إلى قياس فاعلية التعليم عن بعد في ظل تعطل الدراسة في المدارس الماليزية بسبب جائحة كورونا وأجريت الدراسة على طلاب كلية الاتصال والدراسات الإعلامية. أظهرت

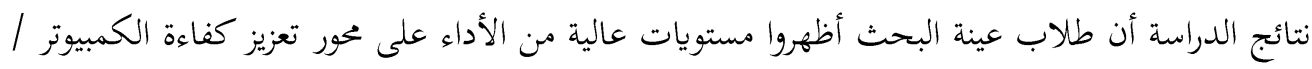
محو الأمية عبر الإنترنت، بينما أظهروا مستويات ضعيفة في محوري التعلم الذاتي. دراسة عساف (2021) بعنوان "تقييم دور الجامعات الفلسطينية في مواجهة الكوارث وإدارة الأزمات في ظل جائحة كورونا" هدفت الدراسة إلى تقييم عينة من طلبة الجامعات الفلسطينية في محافظات غزة ودورهم

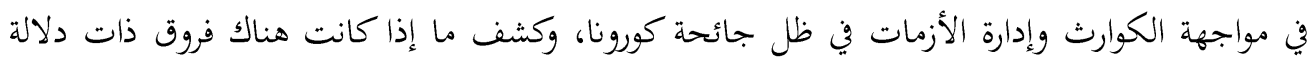
إحصائية عند مستوى (0.05) بين متوسطي درجات. تقييم أعضاء العينة لهذا الدور بسبب المتغيرات (الجنس، الكلية) ولتحقيق ذلك اتبع الباحث المنهج الوصفي التحليلي. أظهرت النتائج، أن درجة تقييم دور

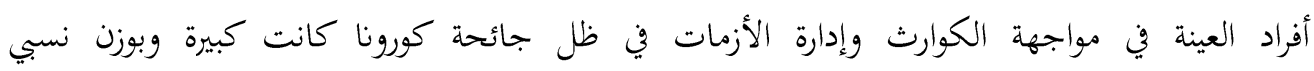

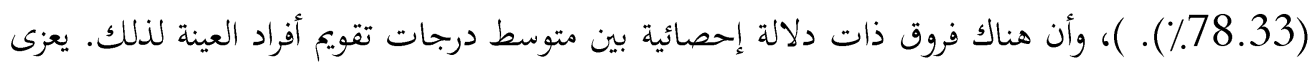

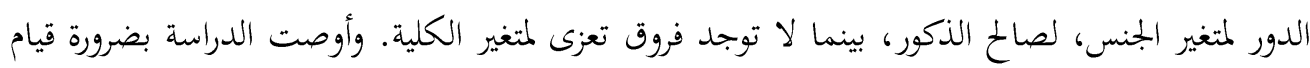

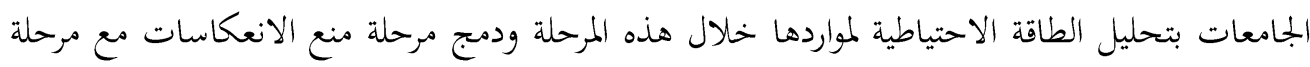
الاستعداد للمواجهة.

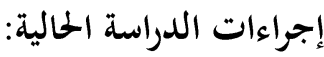

تم الاطلاع على الدراسات السابقة بموضوع الدراسة بغرض الاستفادة منها في إعداد البرنامج الإرشادي من

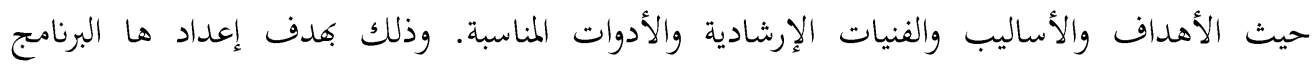
الإرشادي المقترح ليصبح ملائم للتطبيق على المجتمع المستهدف بهن. سيتم في الفقرات الآتية عرض البرنامج الإرشادي المقترح، التي تمخضت عنه نتائج هذه الدراسة المتعلقة بمكونات البرنامج الإرشادي المقترح المناسب الذي يصلح للتدريب على مهارات الذاكرة لطلبة التعليم

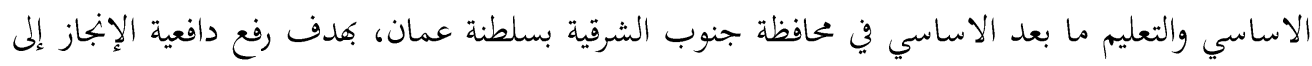
الحد الذي يسمح لهم برفع مستوياتم التحصيلية. 


\section{مجتمع الدراسة المناسب لتطبيق البرنامج:}

هذا البرنامج الإرشادي المقترح يناسب جميع الطلبة التعليم الاساسي والتعليم ما بعد الاساسي في محافظة

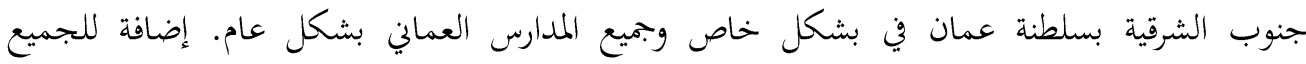
الججتمعات التي تتشابه مع هذا المجتمع مثل طلبة الكليات، كما من الممكن مناسبة هذا البرنامج لطلبة مرحلة التعليم الأساسي في سلطنة عمان وذلك بهدف رفع مستوى تحصيلهم.

البرنامج الإرشادي المقترح لرفع دافعية الإنجاز لدى الطلبة التعليم الاساسي والتعليم ما بعد الاساسي في محافظة جنوب الشرقية بسلطنة عمان

\section{الإطار العام للبرنامج الإرشادي المقتزح:}

قام الباحث ببناء الصيغة (المقترحة) لهذا البرنامج الإرشادي بالاستناد إلى دراسات سابقة التي حاولت بناء برامج إرشادية لرفع المستويات التحصيلية للطلبة والأدب النظري الذي كتب حول المستويات التحصيلية وقد استفاد الباحث بشكل أساسي من دراسة أبو علياء (1993)، ودراسة السوالمة (2001)، ودراسة الطراونة (2005).

\section{أولا: التعريف بالبرنامج الإرشادي المقترح:}

يقوم الباحث بإعداد برنامج مقترح يهدف هذا البرنامج الإرشادي المقترح إلى رفع المستويات التحصيلية لدى الطلبة التعليم الاساسي والتعليم ما بعد الاساسي في محافظة جنوب الشرقية بسلطنة عمان ويتكون البرنامج الإرشادي في المقترحة من (10) جلسات، لكل جلسة أهداف وزمن محدد ومحتوى للجلسة وأساليب وفنيات مستمدة من الإطار النظري للبرنامج.

ثانيا: الأهداف العامة للبرنامج الإرشادي المقتزح:

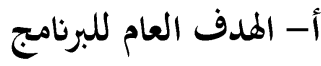

يهدف البرنامج إلى رفع المستويات التحصيلية للطلبة التعليم الاساسي والتعليم ما بعد الاساسي في محافظة

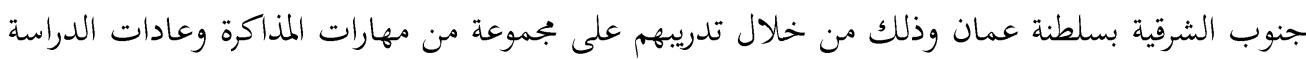

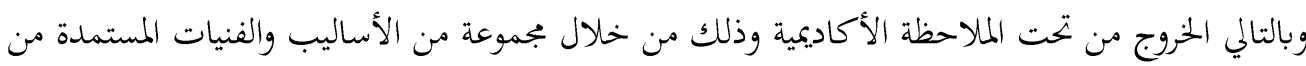
النظريات المعرفية. 
يشير الدوسري (1985) إلى أن البرنامج مخطط منظم على أسس عملية سليمة، ويتكون من مجموعة من الخدمات الإرشادية المباشرة وغير المباشرة، تقدم هذه الخدمات لجميع من تضمهم المدرسة وذلك لتحقيق النمو السوي، والتوافق النفسي والاجتماعي والمهني(18).

\section{ثالثا: محتوى البرنامج الإرشادي:}

يحتوى البرنامج الإرشادي المقترح على (15) جلسة إرشادية جماعية، وتم تحديد الجلسات بحيث يكون لكل

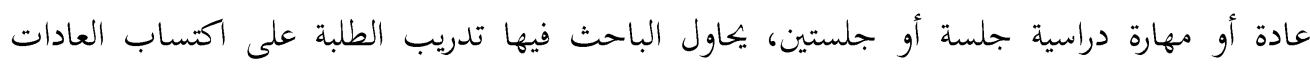
والمهارات الدراسية الصحيحة التي قد تساعد في رفع المستوى التحصيلي للطالب. وتحتوى كل النماذج والأمثلة التي تتضمن مواقف تعليمية يتعلم منها الطالب العادات والمهارات الدراسية الصححيحة.

\section{رابعا: عدد الجلسات ومدتما:}

يتكون البرنامج الإرشادي المقترح الحالي من (10) جلسات إرشادية، سوف تتراوح مدة كل جلسة من (15) دقيقة إلى (25) دقيقة وذلك حسب طبيعة ونوع كل جلسة بواقع جلستين في الأسبوع ولمدة (8) إنسات

\section{خامسا: مخطط الجلسة الإرشادية:}

تحتوى كل جلسة إرشادية من جلسات البرنامج الإرشادي المقترح على: عنوان الجلسة، الأهداف المراد

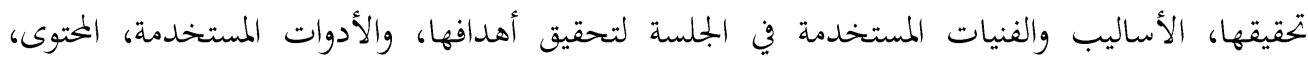

$$
\text { الإجراءات، والواجب البيتي (إن وجد). }
$$

\section{سادسا: محتوى الجلسة الإرشادية:}

تحتوى الجلسة الإرشادية للبرنامج الإرشادي المقترح في بدايتها بالترحيب بأعضاء المجموعة الإرشادية ومناقشة

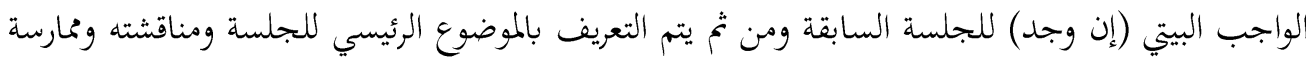

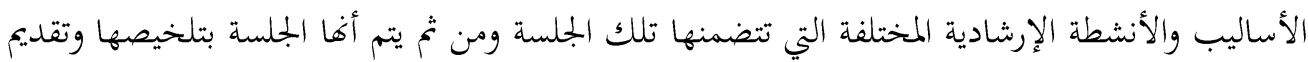

$$
\text { الواجب البيتي (إن وجد). }
$$


مثال على الجلسات الارشادية: - مات

البحلسة الأولى:

\section{العنوان: العلاقة الإرشادية}

أولا: الأهداف: يتوقع من كل مشارك من خلال هذه الجلسة الإرشادية أن: أ. يقوم بالتعرف على المشاركين بشكل مناسب.

ب. يعبر عن تحقيق درجة أفضل من الثقة والألفة والتقبل بينه وبين المرشد وأعضاء الجماعة الإرشادية. ت. توضيح طبيعة البرنامج وأهدافه والواجبات والتدريبات المطلوبة من المجموعة ولئهن الإرشادية، وتعلم قواعد السلوك الجيد.

ث. الاتفاق على منهجية العمل والمواعيد والالتزام على المواظبة في الوقت المحدد. ج. توضيح فكرة مفهوم الإرشاد النفسي الجماعي. ح. تصحيح التوقعات غير الصحيحة للمجموعة الإرشادية، حول الفائدة المرجوة من البرنامج الإرشادي.

ثانيا: الأساليب الإرشادية: المناقشة، الحوار، المحاضرة

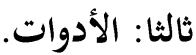
- نموذج خريطة الحياة - بطاقة قواعد السلوك مرك - منوذج لعقد اتفاق

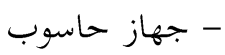
- خدمة انترنت رابعا: الإجراءات.

أ. يستقبل المرشد المجموعة الإرشادية ويرحب بكم ويشكرهم لحضورهم وقبولهم المشاركة. ب. يقوم المرشد بالتعريف على نفسه وطبيعة عمله وسنه ومكان إقامته وأمنيته.

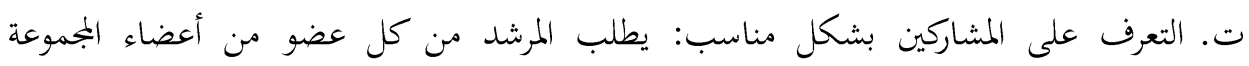
الإرشادية ملء نموذج لخريطة الحياة، ومن ثم يطلب من كل عضو قراء الخريطة التي قام بتبعيتها إمام المجموعة الإرشادية. 
ث. التعبير عن تحقيق درجة أفضل من الثقة والألفة والتقبل بينه وبين المرشد وأعضاء الجماعة

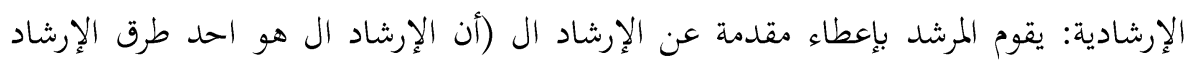

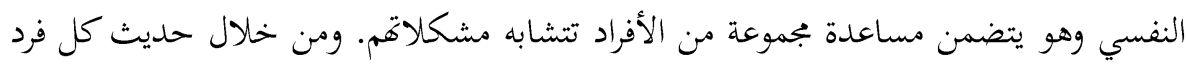

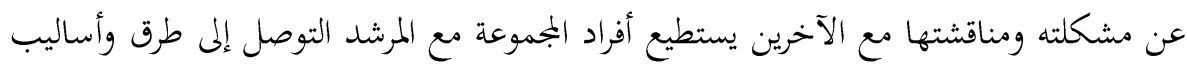

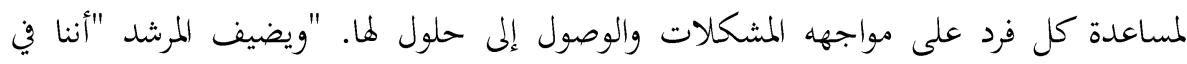

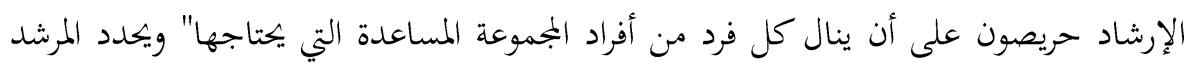

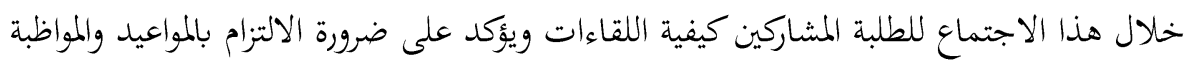
على حضور الاجتماعات الإرشادية وضرورة تنفيذ ما يتفق عليه أفراد المجموعة من تدريبات تتعلق بمهارات الدراسة. ج. توضيح طبيعة البرنامج وأهدافه والواجبات والتدريبات المطلوبة من المجموعة الإرشادية وتعلم قواعد السلوك الجيد. يقوم المرشد بالتعريف عن طبيعة البرنامج وذلك على النحو التالي:

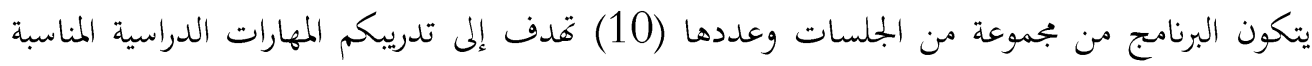
والعادات الدراسية، والتي لها اثر في توافق الطالب الأكاديمي وبالتالي توثر على تحصيله الدراسي. وإن تعاون

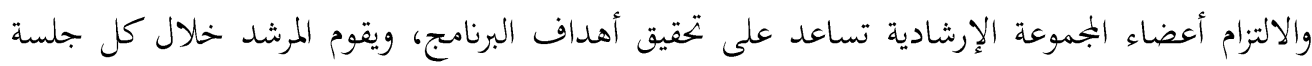

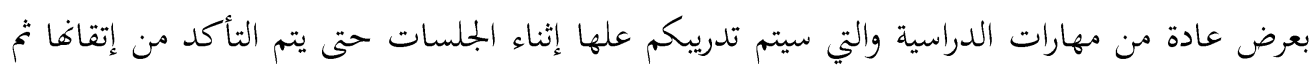
يعطى في نهاية كل جلسة واجب بيتي يتعلق بموضوع الجلسة والمفروض من جميع أعضاء الجمموعة الإرشادية القيام بالواجب ألبيتي الذي سوف تتم مناقشته في الجلسة التالية. ح. يقوم المرشد بتوزيع بطاقة قواعد السلوك الجيد، ويطلب من أعضاء المجموعة الإرشادية قراءتا قراءة جيدة والتعليق عليها. خ. الاتفاق على منهجية العمل والمواعيد والالتزام على المواظبة في الوقت المحدد: يقوم المرشد بالتأكيد

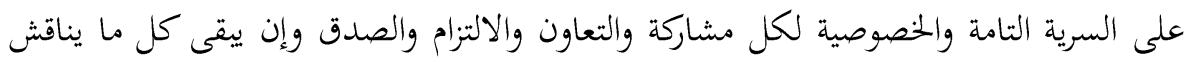
أثناء الجلسة في الجلسة ولا يتحدث عنه مع الآخرين. يطلب المرشد من أعضاء المجموعة

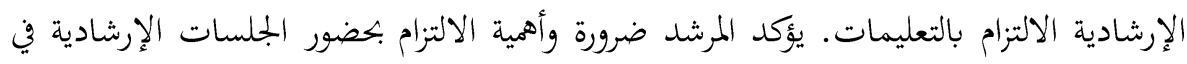
موعدها المحدد وذلك حسب الاتفاق. د. توضيح فكرة مفهوم الإرشاد النفسي الجماعي: يوضح المرشد مفهوم الإرشاد النفسي وأهميته ومبرراته. 
ذ. تصحيح التوقعات غير الصحيحة لأعضاء المجموعة الإرشادية حول الفائدة المرجوة من البرنامج الإرشادي. يقوم المرشد بفتح باب النقاش مع أعضاء المجموعة الإرشادية للتعرف على توقعات إنساء

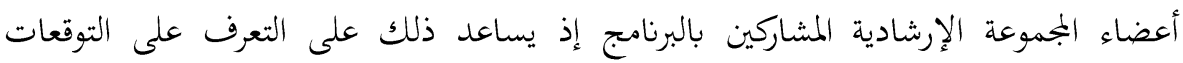
الخاطئة وتصحيحها في مقابل تثبيت التوقعات الواقعية الصحيحة كذلك تعريف المرشد أعضاء المجموعة الإرشادية المشاركين بالتوقعات الممكنة من الإرشاد ال وما هو متوقع منهم من تفاعل ونشاط داخل المجموعة كذلك يساعد على خلق الجو الملائم للإرشاد ال وتحقيق الفائدة المرجوة منه.

$$
\text { الواجب البيتي: (اكتب انطباعك عن الجلسة التي تمت اليوم) }
$$

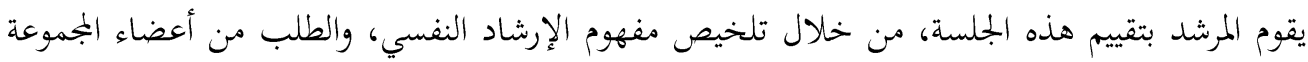
التحدث بما شعر فيه خلال الجلسة، وبيان الرضا منهم والتقبل من كافة أعضاء الجمموعة الإرشادية. ويلاحظ المرشد تعبيرات وجوه الجميع اثنا الجلسة وعندما يغادرون وعلامات الرضا أو عدم الرضا ويقيم مدى حماسهم للقاء في الجلسة التالية.

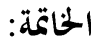

كرس المقال لتصميم برنامج إرشاد مقترح، لرفع دافعية الإنجاز في ضوء جائحة كرونا (كوفيد-19)،

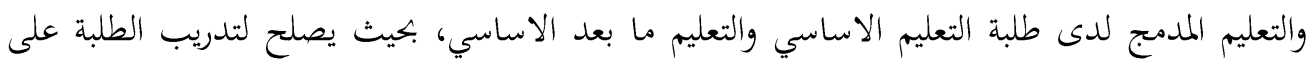
العادات والمهارات الدراسية، التي تساهم في رفع الدافعية. ركزّ برنامج الإرشاد المكون من (10) جلسات إرشادية، على اكتساب مهارات: إدارة الوقت اثناء المحاضرة، تدوين الملاحظات اثناء المحاضرة، تذكر ونسيان كيفية مراجعة الدورات، كيفية التحضير للامتحان،

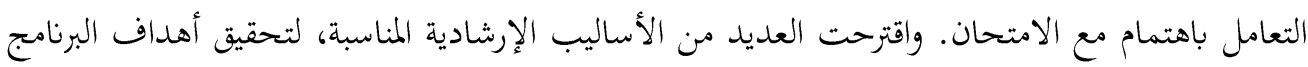
التجريبي، الذي يمثل: حوارات، مناظرة، نمذجة، تعليم تعاوني، عصف ذهني، تغذية راجعة، إدارة الطوارئ، الواجبات المنزلية، التعليمات، القراءة الصامتة، الاسترخاء.

إبراهيم، عبد الرزاق محمود. (2020). معوقات التعليم عن بعد في الجامعة من وجهة نظر اعضاء هيئة الثدريس. مجلة دراسات في العلوم الانسانية المصادر:

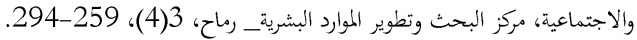

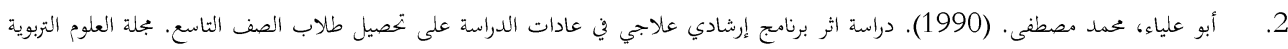

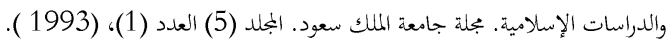




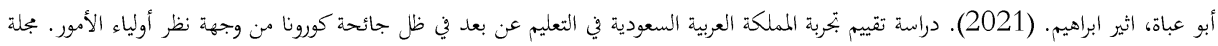

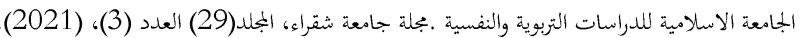

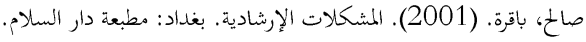

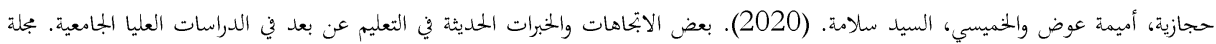
.4

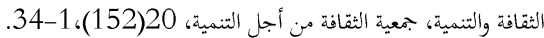

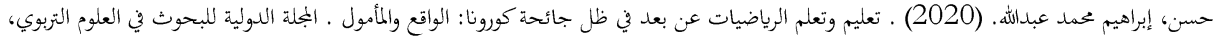

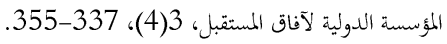

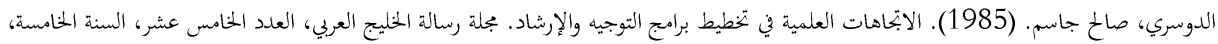
مكتب التربية العربي لدول الخليج، الرياض.

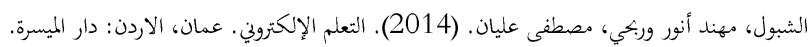

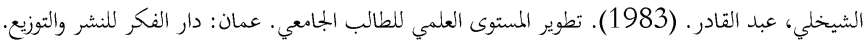

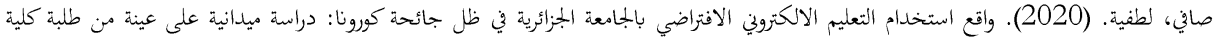
.10

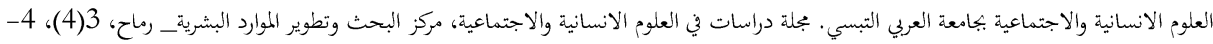

الطراونة، نايف سالم. (2005). أثر برنامج إرشاد عقلاني-انفعالي مصريف في تحسين مستوى دافعية الإنجاز وفاعلية الذات المدركة والمستوى التحصيلي

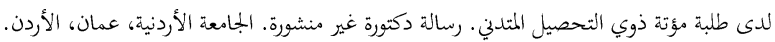

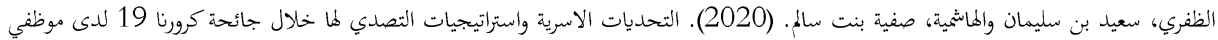

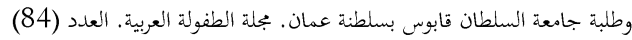

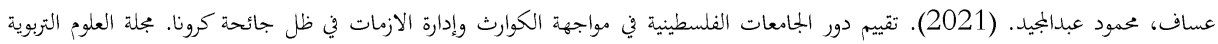

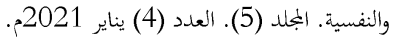

علاء الدين، جهاد محمود. (2005). التحصيل الأكاديمي والعصابية لدى عينة طلبة الجامعة الهاشية، مجلة العلوم التربوية والنفسية، العدد (4)، المجلد .14 . 2005 (4)

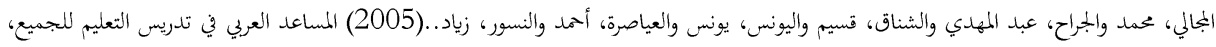
دليل المدرب، وزارة التربية والتعليه، عمان الأردن.

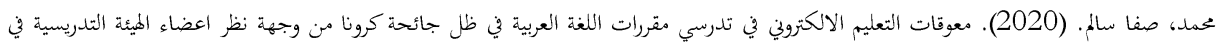
.16

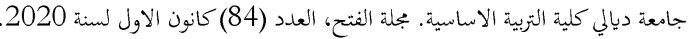

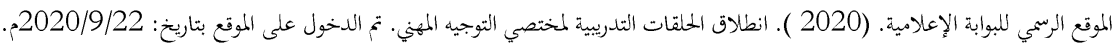

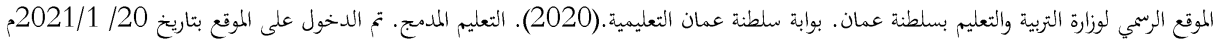

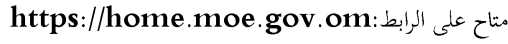

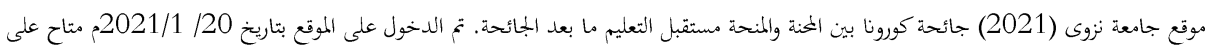

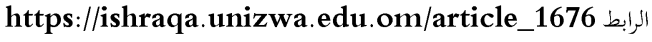

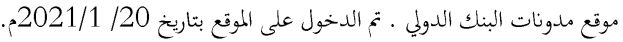




$$
\begin{aligned}
& \text { (6) جامعة نزوى، } 2021 .
\end{aligned}
$$

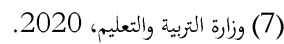

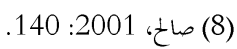

$$
\begin{aligned}
& \text { (9) البنك الدولي، (10) صالح، } 2021 . \\
& \text { (10) البوابة التعليمية، } 2020 \text { (11) } \\
& \text { (11) وزارة التربية والتعليم، } 2020 \text { (10) اليوابة التليه، } 2020 \\
& \text { (12) وزارة التربية، وزارة التية والعليه، } 2020 . \\
& \text { (13) حجازية، الحميسى، وزارة، الرية، } 2020 \text { (12) } \\
& \text { (14) وزارة التربية، } 2020 \text { (13) البحاية البدي، } \\
& \text { (15) البوابة التعليمية، } 2020 \text { (15) الترية، }
\end{aligned}
$$

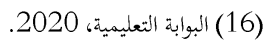

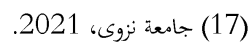

$$
\begin{aligned}
& \text { (18) الدوسري، 1985: صاعن نري، } 238 .
\end{aligned}
$$

